\title{
Review of: "Fungal Spores in Insect Trapping Fluids: Simultaneous Sampling for Insects and Pathogens"
}

\author{
Kentaro Hosaka
}

Potential competing interests: The author(s) declared that no potential competing interests exist.

The manuscript by Berube et al. demonstrated intriguing findings by comparing two methods for collecting spores of forest pathogens. It is particularly informative that Lindgren traps, which have not originally been designed to collect aerial spores, effectively captured diverse range of pathogens. However, because of some technical difficulties and inappropriate experimental settings, I do not think the results from two trapping methods are directly comparable. My major criticisms are summarized below.

(1) Differences in surface area

As indicated by the authors in Discussion, the surface areas of ASC and TF are significantly different. Furthermore, these differences are even more amplified because trapping fluids of TF were filtered and therefore highly concentrated. It is obvious that extracting DNA from the $6 \mathrm{~mm}$ disks, therefore, have significantly different circumstances. Unless some corrected values were presented, I do not think the differences in species diversity between two methods can be compared.

\section{(2) Sampling height}

Sampling by TF was performed at 30-50 cm above the ground whereas ASC method was done $2 \mathrm{~m}$ above ground. Although the differences in height were only less than $2 \mathrm{~m}$, it must have had a significant impact on species richness. Both species richness and spore density in forest air can be significantly different from ground surface to tree canopy. There are a quite a few studies showing vertical distribution of aerial spores and the densities can be very different at every $30 \mathrm{~cm}$ or so. Some comparative results based on different height settings from either method should be presented.

\section{(3) Contamination}

Because TF method was not intended for collecting aerial spores, the risk of contamination should be explained. For example, have the same traps been used previously? Can the traps be sterilized (by using bleach, UV, etc, for example)? Have the traps been stored in forest pathology labs?

(4) Cost and labor

Because of the aforementioned problems, I do not think the results from this study should be interpreted as the differences in efficiency of two collecting methods. Probably the most obvious advantage of using TF 
method is that the traps are being widely used and many samples can be obtained by collaborating with entomologists. This advantage should be emphasized more, but at the same time, some potential disadvantages, such as contamination risk, should also be explained.

Without answering these issues, I do not think this manuscript should be published in this journal. 